\title{
Spinal Tanycytic Ependymoma in Four Korean Patients: Case Series
}

\author{
Jae Yon Choi, Keung Nyun Kim, Dong Ah Shin, Yoon Ha, Do Heum Yoon, Seong Yi \\ Department of Neurosurgery, Spine and Spinal Cord Institute, Severance Hospital, Yonsei University College of Medicine, \\ Seoul, Republic of Korea
}

Corresponding author:

Seong Yi

Department of Neurosurgery,

Spine and Spinal Cord Institute

Severance Hospital, Yonsei

University College of Medicine,

50-1 Yonsei-ro, Seodaemun-gu,

Seoul 03722, Republic of Korea

Tel: +82-2-2228-2174

Fax: +82-2-393-9979

E-mail: sl.sevspine@gmail.com

Received: July 17, 2018

Revised: August 13, 2018

Accepted: August 21, 2018
Tanycytic ependymoma (TE) is a rare variant of ependymoma usually arising in the intramedullary spine found usually on cervical and mid-thoracic level. Herein, we report our experience of treating rare cases of TE. Four patients were studied by retrospective analysis. A pathologist analyzed immunohistochemical results for diagnosis, and postoperative neurologic examination and magnetic resonance imaging (MRI) were followed up. In preoperative MRI study, the tumors were enhanced on T1-weighted imaging which showed well defined lesion. We thought that our patient's tumors were schwannoma. However, spindle cell and immunohistochemical staining of the permanent section revealed strong immunoreactivity for glial fibrillary acidic protein, confirming that tumors were TE. Postoperative neurological symptoms are almost absent. The long-term clinical symptoms for TEs is the same or slightly better than that for other ependymoma subtypes. Diagnosis of TE is challenging as the morphology of the lesions resemble those found in schwannoma and astrocytomas. Differential diagnosis is extremely important for management and prognosis and differentiation of TEs from astrocytomas and schwannomas, although a correct histological diagnosis may be difficult. However, Immunohistochemical staining may be helpful in differential diagnosis.

Key Words: Astrocytoma; Ependymoma; Neurilemmoma

\section{INTRODUCTION}

Ependymoma is a glial tumor that arises from the ependyma, a tissue of the central nervous system. Ependymomas generally present as centrally situated intramedullary tumors within the thoracic/cervico-thoracic cord ${ }^{7}$. Ependymal cells spring from primitive progenitor, the ependymoglia cell, also called "tanycyte". On tracing ependymal and progenital development, transitional types of ependymomas exist. On the World Health Organization (WHO) classification, ependymomas are divided in three major groups: subependymoma (WHO grade I), myxopapillary ependymoma (WHO grade I), ependymoma (WHO grade II), and anaplastic ependymoma (WHO grade III). Conventional (WHO grade II) ependymomas, includes the tanycytic variant.

Tanycytic ependymoma (TE) is a WHO grade II tumor with histological characteristics distinct from the typical features of commonly encountered ependymomas ${ }^{4}$. TE is one of the transitional types of ependymoma ${ }^{2}$. The most common symptoms of TE is the gradual deterioration of motor functions and/or sensory disturbances, such as heaviness, numbness, and weakness of the low back and lower extremities including the waist (paraparesis) accompanied with gait disturbance and urinary incontinence. TE has specific molecular structure. The classic ependymal rosettes and perivascular pseudorosettes of ependymoma are replaced by more fibrillar, spindle cells of tanycyte. The cellular appearance and arrangement may mimic other tumors such as schwannoma and astrocytoma, which makes the diagnosis challenging and hence warrants further evaluation. Since the first case of Friede and Pollak ${ }^{2)}$ was reported in 1978 , only 34 cases have been reported by 2016. Herein, we present four cases (Table 1) of rare ependymoma, TE, which were initially thought to be schwannoma or astrocytoma.

\section{CASE REPORTS}

\section{Case 1}

A 45-year-old man in good health had been having issues with pain in both lower legs and constipation since December 2014. He went to a local clinic and had a magnetic resonance imaging (MRI) done to rule out spinal stenosis. He was referred to our neurosurgery clinic after MRI of the lumbar spine which revealed an extramedullary lesion at the $\mathrm{L} 3$ level. His neurologic examination showed that he had visual analog scale 8 of pain on both legs involving the posterior thigh and calves along 

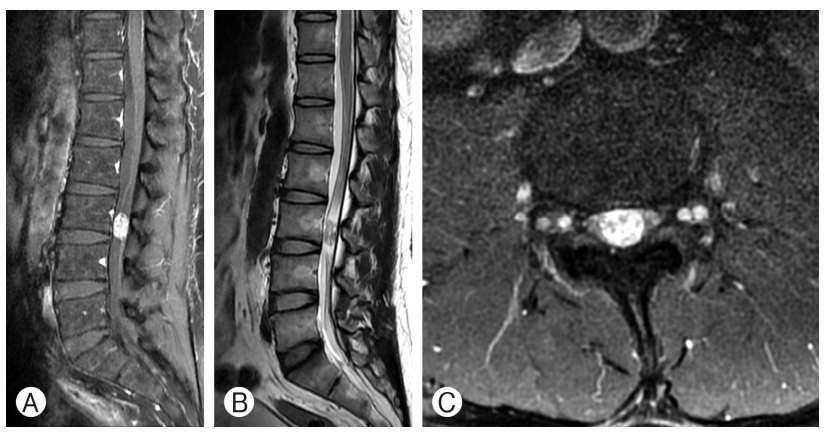

Fig. 1. (A) Sagittal T1-weighted image (M) with gadolinium enhancement showing an avidly enhancing lesion with surrounding cord edema. (B) Sagittal T2-W of the lumbar spine with an isointense lesion at L3 with surrounding T2 hyperintensity suggestive of edema. (C) Axial T1 $\mathrm{W}$ with gadolinium enhancement revealing an extramedullary homogeneously enhancing lesion. the L4-L5 dermatomal distributions. The patient also noted bowel dysfunction. There was no evidence of abnormal reflexes and motor weakness. On MRI, the tumor measured $1.0 \times 1.1 \times$ $1.8 \mathrm{~cm}$, which was at the L3 level. It was an oval-shaped intradural extramedullary filum terminale mass lesion, avidly enhancing on T1-weighted imaging (WI) with mixed intensity on T2-WI (Fig. 1A-C). A laminectomy at L3 was performed. Fusiform-shaped spinal cord tumor that originated from $\mathrm{L} 5$ or $\mathrm{S} 1$ on the right side was exposed. To preserve the nerve root, sub-capsular dissection and tumor removal were done. The tumor capsule with nerve root was preserved (Fig. 2A-C). Microscopically, the tumor was composed of spindle cells with a palisading pattern that resembles verocay bodies of schwannoma and clear cells in some areas (Fig. 3A). Moreover, prominent hyaline blood vessels, macrophages, and few giant cells were detected (Fig. 3B). The tumor cells where strongly and uniformly
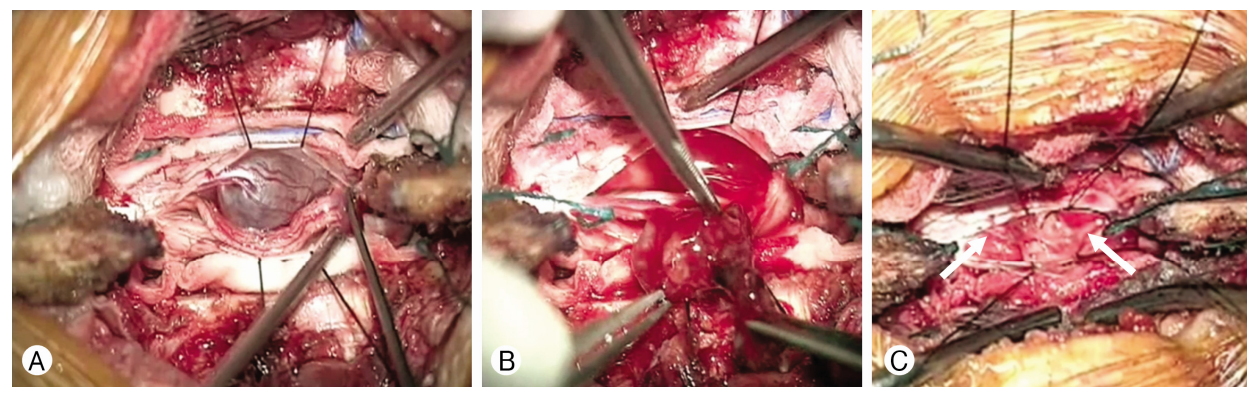

Fig. 2. (A) Fusiform-shaped spinal cord tumor which originated from L5 or S1 at the right side was exposed. (B) Sub-capsular dissection and tumor removal were done. (C) Tumor capsule with nerve root was preserved (arrow).

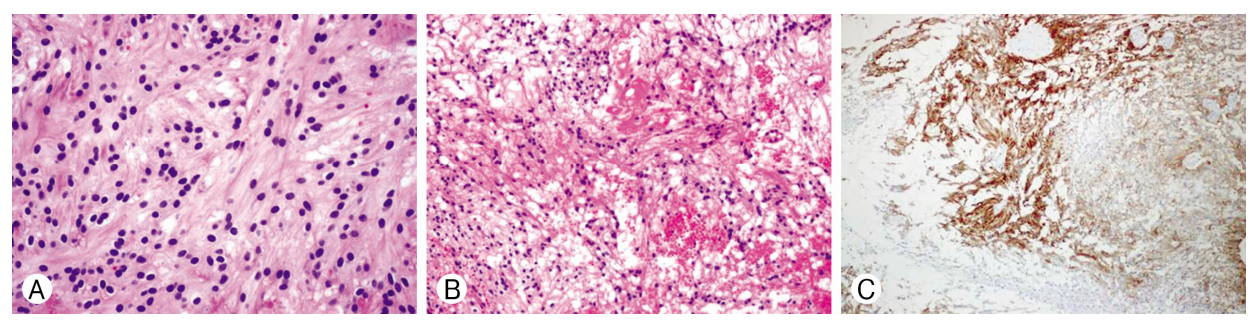

Fig. 3. (A) Histological section showing spindle cell neoplasm of moderate cellularity arranged in interlacing fascicles. Hyalinized blood vessels and foci of hemosiderin pigment deposits are present. (B) Histological section showing neoplastic cells with oval to elongated nuclei with speckled chromatin and small nucleoli. (C) Glial fribillary acidic protein immunohistochemical staining preferentially reacts with fibrillary-appearing cellular elements within tanycytic ependymoma.

Table 1. Clinical presentation and prognosis of four patients

\begin{tabular}{ccccllll}
\hline \hline Patient & Sex & Age & \multicolumn{1}{c}{ Symptom } & Affected level & \multicolumn{1}{c}{ Location } & Treatment & Progression-free period \\
\hline 1 & M & 45 & Leg pain & L3 & Extramedullary & GTR & No recurrence (4 years) \\
2 & F & 44 & Arm numbness & C5 & Intradural & GTR & No recurrence (8 years) \\
3 & F & 59 & Posterior neck pain & C5 & Intradural & GTR & No recurrence (7 years) \\
4 & F & 40 & Lower back pain & L2-L3 & Extramedullary & GTR & No recurrence (4 years)
\end{tabular}

M: male; F: female; GTR: gross total removal. 
positive for glial fribillary acidic protein (GFAP) (Fig. 3C). These findings support the diagnosis of TE. Because gross tumor removal (GTR) of an ependymoma was achieved, no adjuvant therapy was implemented. The patient's postoperative course
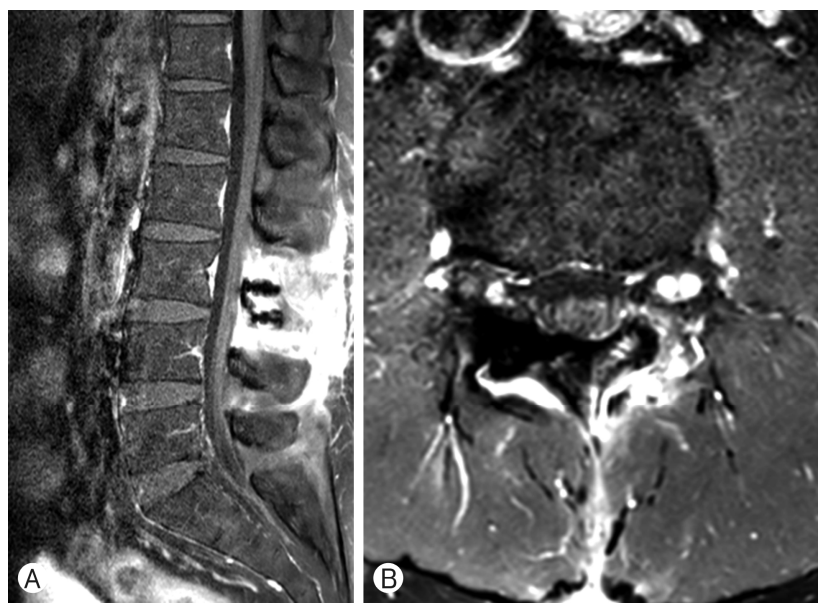

Fig. 4. (A, B) Sagittal and axial gadolinium enhanced $\mathrm{T} 1$-weighted images of the lumbar spine after L3 laminectomies and tumor resection demonstrating gross tumor removal with no residual enhancement. was uneventful. He experienced improvement in his both lower extremity pain. Postoperative MRI showed complete resection with no evidence for recurrence (Fig. 4A, B). Four years after surgery, the patient is free from recurrence.

\section{Case 2}

A 44-year-old woman with no medical problem came to visit us for numbness and tingling sensation in right forearm since 2009 . On MRI, the tumor measured $3.0 \times 1.2 \times 1.6 \mathrm{~cm}$, which was at the C5 level. It was enhancing on T1-WI (Fig. 5A, B). C5 laminectomy was performed, and GTR was done. Microscopically, the tumor was diagnosed with TE. After the surgery, no adjuvant therapy was planned. Postoperative MRI showed complete resection (Fig. 5C, D). Eight years after surgery, the patient has no evidence of recurrence.

\section{Case 3}

A 59-year-old woman with old tuberculosis and major depressive disorder was referred for right arm pain and myelopathy. She had no motor weakness but hypoesthesia at the right arm.
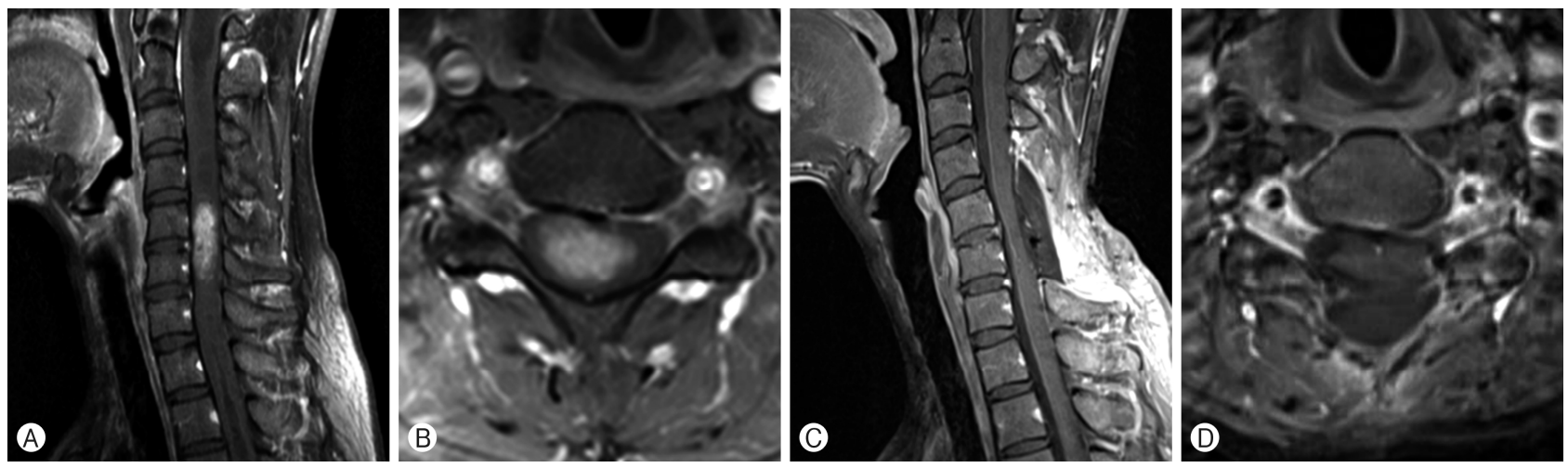

Fig. 5. Pre- and postoperative enhanced $\mathrm{Tl}$-weighted magnetic resonance imaging of case 2. (A) Case 2, preoperative, sagittal. (B) Case 2, preoperative, axial. (C) Case 2, postoperative, sagittal. (D) Case 2, postoperative, axial.
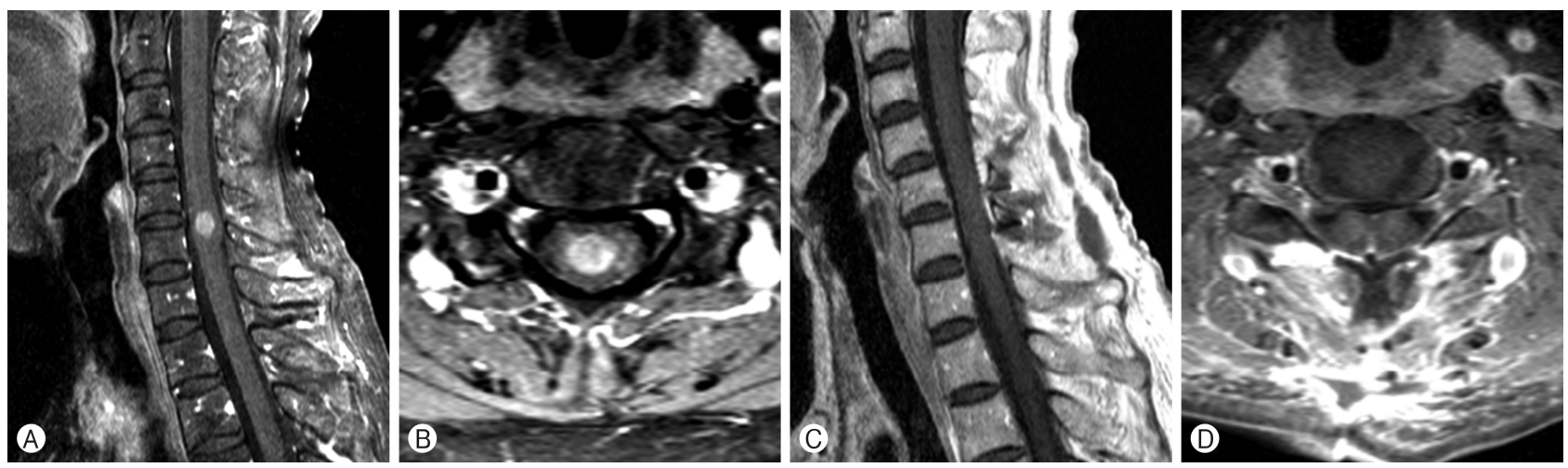

Fig. 6. Pre- and postoperative enhanced T1-weighted magnetic resonance imaging of case 3. (A) Case 3, preoperative, sagittal. (B) Case 3, preoperative, axial. (C) Case 3, postoperative, sagittal. (D) Case 3, postoperative, axial. 

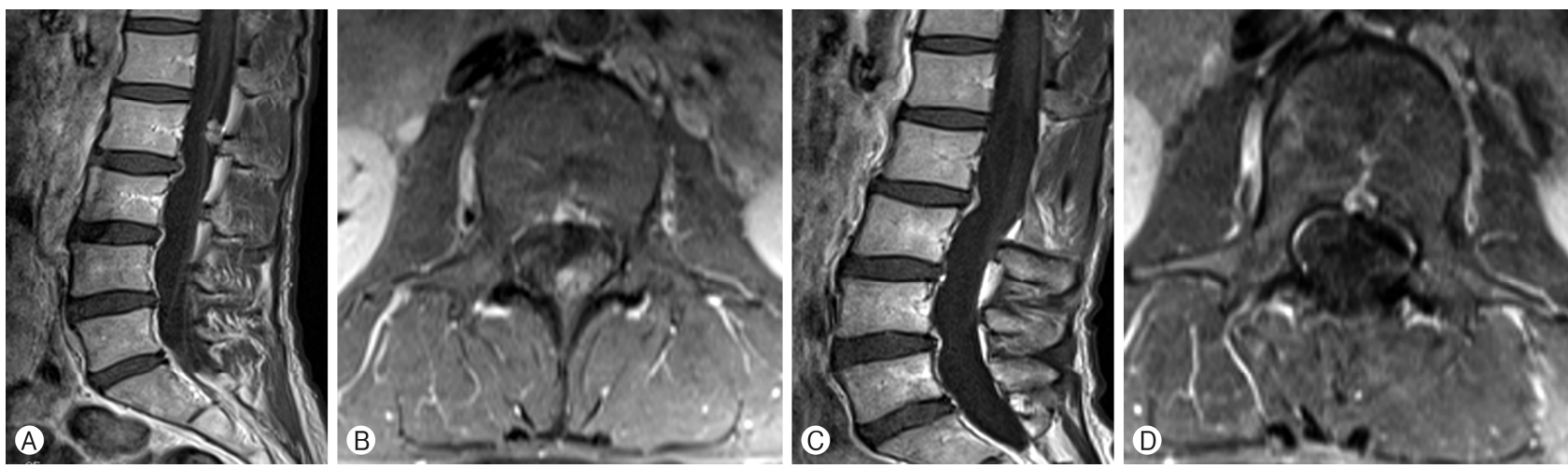

Fig. 7. Pre- and postoperative enhanced T1-weighted magnetic resonance imaging of case 4. (A) Case 4, preoperative, sagittal. (B) Case 4, preoperative, axial. (C) Case 4, postoperative, sagittal. (D) Case 4, postoperative, axial.

Table 2. Comparison of staining methods in schwannoma, pilocytic astrocytoma, and tanycytic ependymoma

\begin{tabular}{llll}
\hline \hline $\begin{array}{c}\text { Histological } \\
\text { stain }\end{array}$ & $\begin{array}{c}\text { Tanycytic } \\
\text { ependymoma }\end{array}$ & Schwannoma & $\begin{array}{c}\text { Pilocytic } \\
\text { astrocytoma }\end{array}$ \\
\hline GFAP & Positive & Negative & Positive \\
Vimentin & Positive & Positive & Negative \\
S-100 & Negative & Positive & Positive
\end{tabular}

Comparison of staining methods in schwannoma, pilocytic astrocytoma, and tanycytic ependymoma.

GFAP: glial fribillary acidic protein.

On MRI, the tumor measured $3.0 \times 1.2 \times 1.6 \mathrm{~cm}$, which was at the C5 level (Fig. 6A, B). C5 laminectomy was performed, and GTR was done. The pathologist confirmed that the tumor was TE. After tumor removal, no adjuvant therapy was performed. Postoperative MRI showed complete resection (Fig. 6C, D). Seven years after surgery, the patient is free from recurrence.

\section{Case 4}

A 41-year-old woman in good health had been having issues with pain on both legs. She had no motor weakness. On MRI, the tumor measured $1.2 \times 0.7 \times 0.7 \mathrm{~cm}$, which was at the L2 level. It was extramedullary mass lesion, enhancing on T1-WI (Fig. 7A, B). L2 laminectomy was performed. The tumor was attached to the filum terminale. The root was sacrificed, and the tumor was completely removed. After the surgery, no neurologic deficit was found. The tumor was confirmed to be TE. Postoperative MRI showed complete resection (Fig. 7C, D). Four years after surgery, the patient is free from recurrence.

\section{DISCUSSION}

TE is of low-to-moderate cellularity characterized by a flow of elongated cells with nuclear pleomorphism. Mitotic figures are usually absent. It has inconspicuous features that are not typical of ependymoma. The distinctive perivascular pseudoro- sette pattern of ependymoma cells is infrequently encountered ${ }^{3)}$. Their nuclei are oval to spindle shaped with evident pleomorphism. The cell processes are not fluffy as with the cellular type of ependymoma ${ }^{8)}$. Radiologically, ependymoma is almost always intramedullary. In our cases the tumor was an extramedullary lesion that also avidly enhanced on T1-WI with gadolinium contrast enhancement. Pilocytic astrocytomas are rarely enhanced $^{11)}$. TE cells may mimic other tumor cells with similar features under light microscopy often lending to a challenging diagnosis ${ }^{3,5,6,9)}$. The spindle appearance of the cells, for instance, is similar to that of schwannoma cells. TE stains positive for GFAP and vimentin but rarely stains for S-100. This helps to differentiate it from schwannoma, which is S-100 positive and GFAP negative. Although pilocytic astrocytoma is GFAP positive, it almost never stains for vimentin, which helps to discriminate it from TE (Table 2) ${ }^{5}$, Pilocytic astrocytoma also presents Rosenthal fibers and eosinophilic granular bodies ${ }^{1,7)}$. Recently, Tao et al. ${ }^{13)}$ reported 40 cases of TE in 2017. Approximately 4000 spinal cord tumors were resected surgically, of which 40 were identified with TE. In terms of prognosis, at a median follow-up of 43 (range, 1-101) months, 1- and 5-year progression-free survival rates were $100.0 \%$ and $97.5 \%$, respectively ${ }^{13}$. The current management is radical excision of spinal TE. It is unlikely to recur if the tumor is completely removed ${ }^{12)}$. Repeat resection or radiation treatment will be recommended in the event of recurrence ${ }^{10)}$. Adjuvant chemotherapy is not recommended $^{14)}$. Through this paper, we reported four cases of TE diagnosed in Korea. Until now, only one case was reported in Korea ${ }^{6}$. Our histologically confirmed four cases will contribute greatly to research on TE in Korea. We also hope these Korean cases will be used as valuable material for interracial and intercontinental study of TE.

\section{CONCLUSION}

Rare cases of spinal TE were presented. TEs are an uncommon fibrillary variant of ependymomas with typical pathological characteristics. A careful and detailed histological inspection 
with utilization of immunohistochemical stains and ultrastructural microscopy may be necessary to distinguish TE from other neoplasms such as schwannoma and pilocytic ependymoma. We believe that this paper could help in the accurate diagnosis and giving appropriate treatment to such population.

\section{CONFLICT OF INTEREST}

No potential conflict of interest relevant to this article was reported.

\section{REFERENCES}

1. Burger PC, Scheithauer BW, Kleinschmidt-DeMasters BK, Ersen A, Rodriguez FJ, Tihan T, et al.: Diagnostic pathology: Neuropathology. Salt Lake City, UT: Amirys Publishing, 2012

2. Friede RL, Pollak A: The cytogenetic basis for classifying ependymomas. J Neuropathol Exp Neurol 37:103-118, 1978

3. Kawano N, Yagishita S, Oka H, Utsuki S, Kobayashi I, Suzuki S, et al.: Spinal tanycytic ependymomas. Acta Neuropathol 101: 43-48, 2001

4. Kleihues P, Cavenee WK: Pathology and genetics of tumours of the nervous system. Lyon, FR: IARC Press, 2000

5. Krisht KM, Schmidt MH: Tanycytic ependymoma: a challenging histological diagnosis. Case Rep Neurol Med 2013:170791, 2013
6. Lim BS, Park SQ, Chang UK, Kim MS: Spinal cord tanycytic ependymoma associated with neurofibromatosis type 2. J Clin Neurosci 17:922-924, 2010

7. Louis DN, Ohgaki H, Wiestler OD, Cavenee WK, Burger PC, Jouvet A, et al.: The 2007 WHO classification of tumours of the central nervous system. Acta Neuropathol 114:97-109, 2007

8. Ng HK: Cytologic features of ependymomas in smear preparations. Acta Cytol 38:331-334, 1994

9. Qian X, Goumnerova LC, De Girolami U, Cibas ES: Cerebrospinal fluid cytology in patients with ependymoma: a bi-institutional retrospective study. Cancer 114:307-314, 2008

10. Ragel BT, Townsend JJ, Arthur AS, Couldwell WT: Intraventricular tanycytic ependymoma: case report and review of the literature. J Neurooncol 71:189-193, 2005

11. Sato K, Kubota T, Ishida M, Handa Y: Spinal tanycytic ependymoma with hematomyelia--case report. Neurol Med Chir (Tokyo) 45:168-171, 2005

12. Sgouros S, Malluci CL, Jackowski A: Spinal ependymomas-the value of postoperative radiotherapy for residual disease control. Br J Neurosurg 10:559-566, 1996

13. Tao X, Hou Z, Hao S, Zhang Q, Wu Z, Zhang J, et al.: The clinical features and surgical outcomes of spinal cord tanycytic ependymomas: A report of 40 cases. World Neurosurg 106:6073, 2017

14. Zacharoulis S, Ashley S, Moreno L, Gentet JC, Massimino M, Frappaz D: Treatment and outcome of children with relapsed ependymoma: a multi-institutional retrospective analysis. Childs Nerv Syst 26:905-911, 2010 